\title{
A Comparative Study on the Outcomes of Mechanically Ventilated COVID-19 vs Non-COVID-19 Patients with Acute Hypoxemic Respiratory Failure
}

\author{
Subhash $\operatorname{Todi}^{1}{ }^{1}$, Sounak Ghosh ${ }^{2}$ (C)
}

\begin{abstract}
Background: Due to the coronavirus disease-2019 (COVID-19) pandemic, there has been a surge of patients requiring mechanical ventilation over a short period of time. The morbidity and mortality outcome in these patients have been variably reported in the published literature. Comparative analyses of ventilated COVID-19 and non-COVID-19 patients during the same time period have been lacking.

Materials and methods: Prospective data for each mechanically ventilated patient was collected from both COVID-19 and non-COVID ICU for a period of 8 months. Their demographic details and disease severity scores were included. Risk-adjusted outcomes across two groups were analyzed using multivariable regression methods.

Results: Crude ICU and hospital mortality were similar in COVID-19- and non-COVID- 19 ventilated groups ( 43.8 vs $40 \%$ and 43.8 vs $41.1 \%$, respectively; $p>0.05$ ). After risk adjustment for the severity of illness by APACHE IV, no significant differences were observed in ICU mortality (OR 1.498; 95\% Cl 0.669-3.327; $p=0.328$ ) and hospital mortality (OR 1.574; 95\% Cl 0.707-3.504; $p=0.267$ ). However, mechanically ventilated COVID-19 patients had increased ICU stay (OR 6.261; 95\% Cl 3.778-8.744; $p<0.001$ ) as well as prolonged ventilatory support (OR 4.358; $95 \% \mathrm{CI}$ $2.910-7.424 ; p<0.001)$ when compared to non-COVID-19 patients.

Conclusion: In mechanically ventilated patients, no significant differences in terms of mortality were noted between COVID-19 and non-COVID-19 patients. Mechanically ventilated COVID-19 patients had longer ICU stay and more number of days on ventilation.

Keywords: Acute respiratory distress syndrome, COVID-19, Mechanical ventilation, Non-COVID-19.

Indian Journal of Critical Care Medicine (2021): 10.5005/jp-journals-10071-24009
\end{abstract}

\section{HIGHLIGHTS}

- Mechanically ventilated coronavirus disease-2019 (COVID-19) patients had longer ICU stay and more number of days on ventilation compared to mechanically ventilated non-COVID-19 patients admitted during the same period.

- There were no significant differences in terms of risk adjusted ICU and hospital mortality between the two groups.

\section{INTRODUCTION}

Coronavirus disease-2019 has created an unprecedented rate of intensive care unit (ICU) admission over a short period of time. ${ }^{1}$ Among those who are hospitalized with COVID-19 infection, up to one-fourth need ICU admission. ${ }^{2}$ Many of these patients require mechanical ventilatory support either on admission or during their ICU stay. ${ }^{3}$ The outcomes of these patients in terms of mortality $\left(4.3^{4}\right.$ $26 \%^{5}$ ) and the length of stay have been variably reported in the literature. Earlier observational studies had reported a much higher adverse outcome in these patients than the present studies. ${ }^{6}$ During the initial period of the COVID-19 pandemic, the threshold for initiating mechanical ventilation was low and early ventilation was the usual practice, ${ }^{7}$ both to avoid contact risk to healthcare workers and to avoid patient self-inflicted lung injury from noninvasive ventilation and high-flow oxygen. Over time, the practice changed and delayed ventilation and prolonged noninvasive oxygen support were adopted ${ }^{8}$ as it was appreciated that with proper precaution the contact infection risk is not high in healthcare workers, and also to avoid the morbidity associated with mechanical ventilation. ${ }^{9}$ This practice change may be responsible for variable outcomes in mechanically ventilated patients. There is a perception among
${ }^{1}$ Critical Care Department, AMRI Hospital, Kolkata, West Bengal, India

${ }^{2}$ Department of Academic and Research, AMRI Hospital, Kolkata, West Bengal, India

Corresponding Author: Subhash Todi, Critical Care Department, AMRI Hospital, Kolkata, West Bengal, India, Phone: +91 9830202040, e-mail: drsubhashtodi@gmail.com

How to cite this article: Todi S, Ghosh S. A Comparative Study on the Outcomes of Mechanically Ventilated COVID-19 vs Non-COVID-19 Patients with Acute Hypoxemic Respiratory Failure. Indian J Crit Care Med 2021;25(12):1377-1381.

Source of support: Nil

Conflict of interest: None

physicians that COVID-19 patients on mechanical ventilation have a worse outcome than their non-COVID-19 counterparts. This has led to the variable practice of initiation of mechanical ventilation in this group of patients. Earlier studies have compared the outcomes of mechanically ventilated COVID-19 patients with a historical non-COVID-19 control arm. ${ }^{10}$ We studied a cohort of COVID-19 and non-COVID-19 ICU patients who were mechanically ventilated during the same time period in order to determine their comparative mortality and length of stay.

\section{Materials and Methods}

\section{Study Population}

The study was conducted in a 227-bed tertiary care private teaching hospital during the time period of 8 months from March

(0) The Author(s). 2021 Open Access This article is distributed under the terms of the Creative Commons Attribution 4.0 International License (https://creativecommons. org/licenses/by-nc/4.0/), which permits unrestricted use, distribution, and non-commercial reproduction in any medium, provided you give appropriate credit to the original author(s) and the source, provide a link to the Creative Commons license, and indicate if changes were made. The Creative Commons Public Domain Dedication waiver (http://creativecommons.org/publicdomain/zero/1.0/) applies to the data made available in this article, unless otherwise stated. 
28,2020 , to November 30,2020 . The study group consisted of all consecutive patients during the study period who were tested positive for SARS-CoV-2 by RT-PCR of respiratory tract specimen and treated in COVID-19 ICU with mechanical ventilator support. This group was compared during the same study period with patients admitted with acute hypoxemic respiratory failure requiring mechanical ventilation who were tested negative for RT-PCR, positive multiplex PCR for other viruses, HRCT chest not compatible with COVID-19, bacterial pneumonia, aspiration pneumonia, and sepsis who were managed in non-COVID-19ICU. Patients who were intubated for elective/emergency surgery, airway protection, and acute exacerbation of chronic obstructive pulmonary disease (COPD), were excluded from the study in nonCOVID-19 ventilated patients.

Dataset was prospectively collected in both the groups and consisted of: (1) patients' demographics, (2) disease severity by APACHE IV, (3) ICU mortality, (4) hospital mortality, (5) ICU length of stay, and (6) hospital length of stay. The mortality and morbidity between these two groups were compared.

Approval of the institutional ethical committee was obtained prior to the study and informed consent was waived due to the observational nature of the study.

\section{Statistical Analyses}

After selecting the study population, variables were tested for normal distribution. Baseline characteristics of the participants were analyzed using mean \pm standard deviation (SD) for continuous variables and percentages for categorical variables. We used the independent sample $t$ test and chi-square test for analyzing the differences among COVID-19 and non-COVID-19 ventilated patients. A multivariable risk-adjusted regression analysis was performed to examine whether COVID-19 infection status is an independent risk factor for study outcomes.

We used IBM SPSS Statistics v22 for all data analyses and twotail $p$ value $<0.05$ has been considered significant.

\section{Results}

\section{Characteristics of Study Population}

A total of 48 COVID-19 patients were mechanically ventilated and 159 non-COVID-19 patients were ventilated during the same study period of 8 months. Of the 159 non-COVID-19 patients, 95 patients with acute hypoxemic respiratory failure were included in the study (Table 1). Sixty-four patients of the non-COVID-19 group were excluded as they were intubated for perioperative reasons, airway protection, and exacerbation of COPD with hypercapnic respiratory failure.

The baseline characteristics across these two groups are shown in Table 2. COVID-19 patients who were on ventilator support were more likely to be younger ( 63.79 vs 67.59 years), male ( 68.8 vs $31.3 \%$ ) than non-COVID-19 ventilated patients. COVID-19 patients on ventilation also had a significantly elevated $\mathrm{C}$-reactive protein (CRP). Moreover, severity scoring (APACHE IV), predicted mortality rate (PMR), and expected ICU length of stay were nonsignificantly lower in the COVID-19 group.

\section{Crude Outcome Analyses between COVID-19 and Non-COVID-19 Groups}

Crude ICU and hospital mortality are nonsignificantly higher in COVID-19 patients than their non-COVID-19 counterparts (43.8 vs $40 \%$ and 43 vs $41.1 \%$, respectively) (Table 3). PMR as per APACHE IV had a lower trend in COVID-19 patients and standardized mortality ratio (SMR) was nonsignificantly higher in COVID-19 patients. PMR is calculated based on a disease category as viral pneumonia which is not specific for COVID-19. Due to this reason, SMR was calculated by dividing the observed mortality rate with the PMR. Thus, the SMR calculation was higher for COVID-19 patients. The expected length of ICU stay as per APACHE IV was similar in both groups. The observed ICU length of stay was significantly higher in COVID-19 patients (13.75 vs 7.67 days) but hospital length of stay was similar in both COVID-19 and non-COVID-19 groups (14.6 vs 12.73 days; $p>0.05)$. The number of days on the ventilator was significantly

Table 1: Diagnosis of the participants included in the non-COVID-19 ventilated group

\begin{tabular}{lc}
\hline Diagnosis & No of patients \\
\hline Bacterial pneumonia & 21 \\
Viral pneumonia & 22 \\
Aspiration pneumonia & 3 \\
Sepsis & 49 \\
\hline
\end{tabular}

Table 2: Characteristics of ventilated COVID-19 patients vs non-COVID-19 patients

\begin{tabular}{|c|c|c|c|}
\hline Variables & Non-COVID-19 $(95 ; 66.43)$ & COVID-19 $(N=48 ; 33.56)$ & $p$ value \\
\hline \multicolumn{4}{|l|}{ Demographics } \\
\hline Age, years & $67.59 \pm 13.15$ & $63.79 \pm 14$ & 0.113 \\
\hline Gender, $N(\%)$ & & & 0.435 \\
\hline - Female & $27(28.4)$ & $15(31.3)$ & \\
\hline - Male & $68(71.6)$ & $33(68.8)$ & \\
\hline Hypertension, $N(\%)$ & $54(57.9)$ & $22(45.8)$ & 0.310 \\
\hline Diabetes, $N(\%)$ & $48(50.52)$ & $18(37.5)$ & 0.228 \\
\hline $\mathrm{SBP}, \mathrm{mm} \mathrm{Hg}$ & $103.95 \pm 26.86$ & $115.74 \pm 27.66$ & $0.020^{*}$ \\
\hline $\mathrm{CRP}, \mathrm{mg} / \mathrm{dL}$ & $118.05 \pm 122.11$ & $164.67 \pm 114.85$ & $0.032^{*}$ \\
\hline APACHE IV & $85.47 \pm 28.29$ & $76.66 \pm 31.36$ & 0.349 \\
\hline PMR & $42.52 \pm 28.86$ & $37.38 \pm 30.99$ & 0.865 \\
\hline Expected ICU length of stay, days & $6.47 \pm 4.56$ & $5.92 \pm 2.04$ & 0.355 \\
\hline
\end{tabular}


Comparisons of the Outcomes of Ventilated COVID vs Non-COVID-19 Patients

Table 3: Differences in outcome variables among COVID-19 and non-COVID-19 ventilated patients

\begin{tabular}{lccc}
\hline Outcome variables & Non-COVID-19 & COVID-19 & p value \\
\hline ICU mortality, N (\%) & $38(40)$ & $21(43.8)$ & 0.400 \\
Hospital mortality, N (\%) & $39(41.1)$ & $21(43.8)$ & 0.455 \\
SMR & 0.91 & 1.17 & \\
Total ICU stay, days & $7.67 \pm 6.70$ & $13.75 \pm 10.11$ & $<0.001^{*}$ \\
Total hospital stay, days & $12.73 \pm 10.73$ & $14.60 \pm 9.40$ & 0.140 \\
Days on ventilation & $6.22 \pm 5.94$ & $10.54 \pm 9.20$ & $0.001^{*}$ \\
Expired patients on ventilation, days & $4.62 \pm 5.58$ & $7.76 \pm 6.64$ & 0.056 \\
Alive patients on ventilation, days & $7.34 \pm 5.78$ & $12.70 \pm 10.40$ & $0.006^{*}$ \\
\hline
\end{tabular}

${ }^{*} \mathrm{ICU}$, intensive care unit; SMR, standardized mortality ratio

Table 4: Association between COVID-19 status and the study outcomes adjusted by APACHE IV

\begin{tabular}{lccc}
\hline Variables & OR & 95\% confidence interval & p value \\
\hline ICU mortality & 1.498 & $0.669-3.327$ & 0.328 \\
Hospital mortality & 1.574 & $0.707-3.504$ & 0.267 \\
ICU stay & 6.261 & $3.778-8.744$ & $<0.001^{*}$ \\
Total hospital stay & 1.764 & $-2.482-6.011$ & 0.413 \\
Days on ventilation & 4.358 & $2.910-7.424$ & $0.005^{*}$ \\
\hline
\end{tabular}

higher in COVID-19 patients (10.54 vs 6.22 days). Ventilator-free days in patients who survived were significantly longer in the COVID-19 group (3.92 vs 1.98 days).

\section{Risk-adjusted Outcome Analyses between COVID-19 and Non-COVID-19 Groups}

In multivariable regression analyses, after adjustment with APACHE IV, significant association was observed in total ICU stay (OR 6.261; 95\% Cl 3.778-8.744; $p<0.001$ ), ventilator-free days for alive patients (OR 2.095; 95\% Cl 0.472-3.718; $p=0.012$ ) and total ventilator days (OR 4.358; 95\% Cl 2.910-7.424; $p=0.005$ ) in COVID19 patients. Importantly, even after risk adjustment, no significant associations were found in total hospital stay, ICU mortality, and total hospital mortality in COVID-19 patients $(p>0.05)$ (Table 4).

\section{Discussion}

In this observational study, a cohort of mechanically ventilated COVID-19 patients was analyzed with regard to their ICU and hospital mortality and length of stay. This cohort was further compared for similar outcome parameters, during the same time period in non-COVID-19 mechanically ventilated patients intubated for acute hypoxemic respiratory failure. Our findings on the demographics of age and gender in COVID-19-infected ventilated patients were similar to the published literature. ${ }^{11}$ We noted that the COVID-19-infected cohort was of a younger age and of male predominance as compared to non-COVID-19 patients. Hypertension was more prevalent in non-COVID-19 ICU patients which may be explained by their relatively older age. The ICU and hospital mortality of ventilated COVID-19 patients of $43.8 \%$ were also consistent with the published literature, ${ }^{12}$ though the literature in this regard is variable with reports of case fatality rate COVID-19 ranging from $12-78 \%$ in various reports with an average of $25-50 \% .{ }^{13,14}$ This variability could be due to a true variation in mortality based on geographical location, resource availability, and variable treatment protocols. This could also be due to different methods of reporting outcomes, with some studies not reporting the ICU outcomes for patients still on mechanical ventilation during the study. ${ }^{15}$ In our cohort, all patients on mechanical ventilation during the study period were analyzed till extubated or expired. A similar approach was adopted in the non-COVID-19 patient group. Moreover, in our analysis, PMR as per APACHE IV was nonsignificantly lower in COVID-19 patients as opposed to SMR which was higher in this group as compared to non-COVID-19 patients. This could be due to the fact that PMR is calculated in the APACHE IV database based on a disease category as "viral pneumonia" which is not specific for COVID-19. And SMR is calculated by dividing the observed mortality rate with the PMR. Thus, the SMR calculation was higher for COVID patients.

The ICU and hospital mortality rates of non-COVID-19 ventilated patients were 40 and $41.1 \%$, respectively which were similar to the published literature mortality of $40-46 \%$ in moderate-to-severe non-COVID-19 ARDS. ${ }^{16}$ Thus, in our study, there was no significant difference in ICU and hospital mortality of $40 \%$ both in crude and risk-adjusted (APACHE IV) analyses during the study time period in COVID-19 and non-COVID-19 mechanically ventilated patients. This observation has clinical applicability as reports of high mortality in some publications of COVID-19 infected mechanically ventilated patient creates an impression of more adverse outcome than their non-COVID-19 counterparts, thus dissuading many physicians from ventilating COVID-19 patients. The literature review did not reveal a similar comparative study performed previously in this cohort of patients during a similar time period. However, there was a trend toward a marginal increase in SMR in COVID-19 patients due to lower PMR. Interestingly, the ICU mortality in the control arm of the RECOVERY study of dexamethasone in mechanically ventilated COVID-19 patients was $41.4 \%$ which was similar to our cohort of COVID-19 ventilated patients; most of whom have received steroids. Dexamethasone arm of the RECOVERY study had a mortality of $29.3 \%$ which is lower than our observed mortality. ${ }^{17}$ 
Total ventilator days and ventilator-free days for alive patients are also important morbidity outcome parameters. In our study, mean ventilator days and ventilator-free days in COVID-19 patients were 10.54 and 3.92 days, respectively which was similar to the published literature. ${ }^{18}$ When compared to their non-COVID-19 counterpart, these durations were much longer, reflecting a much larger resource utilization of COVID-19 patients. This trend also reflects not only increased duration of ventilation in the COVID-19 group but also prolonged post-ventilation ICU stay in this group.

ICU and hospital length of stay are other outcome parameters for resource allocation optimization. In our study, for COVID-19 patients, these were 13.75 and 14.60 days, respectively. Expected ICU length of stay as per APACHE IV was similar in both the groups, thereby increasing the importance of the observed increased length of stay in the COVID-19 ventilated group. When compared to non-COVID-19 counterpart, ICU length of stay was significantly longer in COVID-19 patients than in the non-COVID-19 group. This was not reflected in the total hospital length of stay between the two cohorts which were similar. This may be due to the early hospital discharge of COVID-19 patients.

The strength of our study is the prospective nature of data collection, the inclusion of all consecutive mechanically ventilated patients in both COVID-19 and non-COVID-19 groups during a similar time period, analysis of patient data till they were either extubated or died. Outcome variables of ICU and hospital mortality, ventilator-free days, and ICU and hospital length of stay are all clinically relevant outcome parameters. Using a risk-adjusted mortality outcome with the severity of illness removes the confounding bias. Most importantly, the comparison of COVID-19 with the non-COVID-19 counterpart of acute hypoxemic respiratory failure is a unique feature of this study. Using a concurrent non-COVID-19 cohort rather than a historical cohort is an important consideration as in the pandemic resource allocation is different and the outcome of mechanically ventilated patients depends on this parameter, thus subjecting both the cohort to a similar resource limitation. Observational studies of this kind give an insight into the outcome of COVID-19 patients when compared to their non-COVID-19 counterparts, thereby diminishing apprehensions of worse outcome in COVID-19 mechanically ventilated patients.

The limitation of this study is single center, a less number of mechanically ventilated COVID-19 patients than their non-COVID-19 counterparts. Due to the small sample size, the power of the study may not be large enough to detect clinically significant mortality differences between the two cohorts.

Future multicentric studies of similar nature with the propensity of matching the prognostic variables in both cohorts with adequate sample size will be important to analyze important outcome variables.

\section{Conclusion}

The comparative study of mechanically ventilated COVID-19 and non-COVID-19 patients with acute hypoxemic respiratory failure on mechanical ventilation during a similar time period had shown a similar risk-adjusted ICU and hospital mortality in these two cohorts. ICU length of stay and ventilator-free days of alive patients were significantly longer in COVID-19 patients.

\section{Author Contribution}

The idea was conceived by Dr S Todi. Data organization and statistical analyses were done by Dr S Ghosh. The manuscript was written and revised by Dr S Todi and Dr S Ghosh. The final version has been approved by both authors.

\section{ORCID}

Subhash Todi (이 https://orcid.org/0000-0003-2306-6080

Sounak Ghosh (1) https://orcid.org/0000-0003-4245-0811

\section{References}

1. Jain V, Yuan JM. Predictive symptoms and comorbidities for severe COVID-19 and intensive care unit admission: a systematic review and meta-analysis. Int J Public Health 2020;65(5):533-546. DOI: 10.1007/ s00038-020-01390-7.

2. Grasselli G, Pesenti A, Cecconi M. Critical care utilization for the COVID-19 outbreak in Lombardy, Italy: early experience and forecast during an emergency response. JAMA 2020;323(16):1545-1546. DOI: 10.1001/jama.2020.4031.

3. Arentz M, Yim E, Klaff L, Lokhandwala S, Riedo FX, Chong M, et al. Characteristics and outcomes of 21 critically ill patients with COVID-19 in Washington State. JAMA 2020;323(16):1612-1614. DOI: 10.1001/jama.2020.4326.

4. Wang D, Hu B, Hu C, Zhu F, Liu X, Zhang J, et al. Clinical characteristics of 138 hospitalized patients with 2019 novel coronavirus-infected pneumonia in Wuhan, China. JAMA 2020;323(11):1061-1069. DOI: 10.1001/jama.2020.1585.

5. Grasselli G, Zangrillo A, Zanella A, Antonelli M, Cabrini L, Castelli A, et al. Baseline characteristics and outcomes of 1591 patients infected with SARS-CoV-2 admitted to ICUs of the Lombardy Region, Italy. JAMA 2020;323(16):1574-1581. DOI: 10.1001/jama.2020.5394.

6. Wang Y, Lu X, Li Y, Chen H, Chen T, Su N, et al. Clinical course and outcomes of 344 intensive care patients with COVID-19. Am J Respir Crit Care Med 2020;201(11):1430-1434. DOI: 10.1164/rccm.2020030736LE.

7. ZarbockA, Kellum JA, Schmidt C, Van Aken H, Wempe C, Pavenstädt H, et al. Effect of early vs delayed initiation of renal replacement therapy on mortality in critically ill patients with acute kidney injury: the ELAIN randomized clinical trial. JAMA 2016;315(20):2190-2199. DOI: 10.1001/jama.2016.5828.

8. Holmen IC, Kent A, Lakritz S, Brickson C, Mastalerz K. Delayed tracheostomy in a patient with prolonged invasive mechanical ventilation due to COVID-19. Cureus 2020;12(6):e8644. DOI: 10.7759/ cureus.8644.

9. Windisch W, Weber-Carstens S, Kluge S, Rossaint R, Welte T, Karagiannidis $C$. Invasive and non-invasive ventilation in patients with COVID-19. Deutsch Arztebl Int 2020;117(31-32):528-533. DOI: 10.3238/arztebl.2020.0528.

10. Sjoding MW, Admon AJ, Saha AK, Kay SG, Brown CA, Co I, et al. Comparing clinical features and outcomes in mechanically ventilated patients with COVID-19 and the acute respiratory distress syndrome. Ann Am Thorac Soc 2021. DOI: 10.1513/AnnalsATS.202008-1076OC.

11. Mohan A, Tiwari P, Bhatnagar S, Patel A, Maurya A, Dar L, et al. Clinicodemographic profile \& hospital outcomes of COVID-19 patients admitted at a tertiary care centre in north India. Indian J Med Res 2020;152(1 \& 2):61-69. DOI: 10.4103/ijmr.IJMR_1788_20.

12. Kang SJ, Jung SI. Age-related morbidity and mortality among patients with COVID-19. Infect Chemother 2020;52(2):154-164. DOI: 10.3947/ ic.2020.52.2.154.

13. Du RH, Liang LR, Yang CQ, Wang W, Cao TZ, Li M, et al. Predictors of mortality for patients with COVID-19 pneumonia caused by SARSCoV-2: a prospective cohort study. Eur Respir J 2020;55(5):2000524. DOI: 10.1183/13993003.00524-2020. 
14. COVID-ICU Group on behalf of the REVA Network and the COVID-ICU Investigators. Clinical characteristics and day-90 outcomes of 4244 critically ill adults with COVID-19: a prospective cohort study. Intensive Care Med 2021;47(1):60-73. DOI: 10.1007/s00134-020-06294-x.

15. Anesi GL, Jablonski J, Harhay MO, Atkins JH, Bajaj J, Baston C, et al. Characteristics, outcomes, and trends of patients with COVID-19related critical illness at a learning health system in the United States. Ann Intern Med 2021;174(5):613-621. DOI: 10.7326/M20-5327.

16. Bellani G, Laffey JG, Pham T, Fan E, Brochard L, Esteban A, et al. Epidemiology, patterns of care, and mortality for patients with acute respiratory distress syndrome in intensive care units in 50 countries. JAMA 2016;315(8):788-800. DOI: 10.1001/jama.2016.0291.

17. Horby P, Lim WS, Emberson JR, Mafham M, Bell JL, Linsell L, et al. Dexamethasone in hospitalized patients with Covid-19. N Engl J Med 2021;384(8):693-704. DOI: 10.1056/NEJMoa2021436.

18. Badr M, De Oliveira B, Abdallah K, Nadeem A, Varghese $Y$, Munde $D$, et al. Effects of methylprednisolone on ventilator-free days in mechanically ventilated patients with acute respiratory distress syndrome and COVID-19: a retrospective study. J Clin Med 2021;10(4):760. DOI: 10.3390/jcm10040760. 